\title{
Indocyanine green angiography of the anterior segment in patients undergoing strabismus surgery
}

Tin K J Chan, Arthur L Rosenbaum, Rajesh Rao, Steven D Schwartz, Pauline Santiago, Dennis Thayer
Department of Ophthalmology, University of California, Los Angeles, CA, USA T K J Chan A L Rosenbaum R Rao

S D Schwartz P Santiago

D Thayer

Department of Ophthalmology, Royal Hallamshire Hospital, University of Sheffield, Sheffield, UK

T K J Chan

Correspondence to: Arthur Rosenbaum, MD, Jules Stein Eye Institute, 100 Stein Plaza, UCLA, Los Angeles, CA 90095-7002, USA

rosenbaum@

jseint.jsei.ucla.edu

Accepted for publication 19 November 2000

\begin{abstract}
Background-Anterior segment imaging using fluorescein angiography is only suitable in lightly pigmented irides as the brown pigmentation of the iris masks fluorescein transmission. Indocyanine green (ICG) angiography has excellent penetration of pigment epithelium and, therefore, has potential application in detecting perfusion changes of dark irides after strabismus surgery.

Methods-A prospective study was conducted on patients older than 15 years undergoing strabismus surgery. A fundus camera was focused on the arteriolar tufts of the pupillary margin and $50 \mathrm{mg}$ of ICG (concentration of $12.5 \mathrm{mg} / \mathrm{ml}$ ) was given intravenously. Images were then obtained at 1 minute intervals of 5 minutes' duration.

Results-45 patients with a mean age of 54.6 years and a mean follow up period of 8.6 weeks were studied. There were 23 patients in the primary surgery group, 11 in the secondary surgery group, and 11 in the staged group. Iris ICG angiograms were successfully performed in all patients. No persistent filling defect was detected in the primary and secondary horizontal recti surgery groups or in the secondary or staged vertical and combined vertical rectus groups 6-8 weeks postoperatively. $57 \%$ of both primary vertical and combined vertical and horizontal groups showed defects in the early postoperative phase. Only three cases demonstrated late perfusion defects in this series.

Conclusion-ICG can detect iris perfusion changes in dark irides after strabismus surgery. Iris reperfusion was achieved in the majority of the cases.

(Br f Ophthalmol 2001;85:214-218)
\end{abstract}

The risk of anterior segment ischaemia (ASI) is increased in complex strabismus procedures and retinal detachment surgery involving encirclement elements. ${ }^{1}$ Anterior segment (AS) imaging using fluorescein angiography
(FA) was first described in 1968 but the brown pigment of the iris masked fluorescein transmission. ${ }^{23}$

Indocyanine green (ICG) is a dye that has a peak absorption at 790-805 $\mathrm{nm}$ and it fluoresces at $825-835 \mathrm{~nm}$. Since only $10 \%$ of the light is absorbed at this infrared range, the dye can be seen through overlying haemorrhage, melanin, fluid, xanthophyll, etc. ${ }^{4}$ With the advent of digitalised imaging, ICG angiography (ICGA) is useful for studying the choroidal vasculature. ${ }^{56}$ ICGA of the iris becomes a potential diagnostic tool because of its ability to fluoresce at a wavelength not blocked by overlying pigment.

\section{Materials and methods}

Using ICGA, the pattern of normal iris circulation was studied. The effect of different types of rectus muscle surgery on the anterior segment circulation was evaluated. Adult patients (at least 21 years of age except one patient aged 15 years) scheduled to undergo strabismus surgery by limbal conjunctival approach were grouped as follows:

- Primary rectus muscle surgery was defined as patients with no previous strabismus surgical history or evidence of rectus muscle surgery

- Secondary surgery was defined as surgery on a previously operated rectus muscle

- Staged rectus muscle surgery was defined as surgery to previously unoperated rectus muscle when other rectus muscle(s) in the same eye had undergone surgery.

The Topcon TRC50 IA fundus camera was used to obtain colour AS photographs. The camera was focused on the arteriolar tufts at the pupil margin. ICG at $12.5 \mathrm{mg} / \mathrm{ml}$ for a total dose of $50 \mathrm{mg}$ was given via intravenous bolus at a rate of $1 \mathrm{ml}$ per second. Images were obtained at approximately 1 or 2 second intervals until the iris circulation achieved maximum brightness. Images were then obtained at 1 minute intervals for 5 minutes, then at 3 minute intervals for a total duration of $30-40$ minutes. Images were saved using the NEC TI-23A video camera on the Topcon Image Net H1024 digital imaging system and stored on an optical disc for analysis. 
Table 1 Type of operation and presence of sectorial delay in early and late postoperative phases

\begin{tabular}{|c|c|c|c|}
\hline Rectus muscle & Number of eyes & $\begin{array}{l}\text { Delay in filling } \\
\text { (early) }\end{array}$ & $\begin{array}{l}\text { Delay in filling } \\
\text { (late) }\end{array}$ \\
\hline \multicolumn{4}{|l|}{ Primary } \\
\hline Horizontal & 9 & None & None \\
\hline 1 Muscle & 4 & & \\
\hline 2 Muscles & 5 & & \\
\hline Vertical & 9 & $6^{\star}$ & 1 \\
\hline 1 Muscle & 4 & & 1 \\
\hline 2 Muscles & 2 & & 0 \\
\hline Vertical and horizontal & 5 & $4 \dagger$ & None \\
\hline \multicolumn{4}{|l|}{ Secondary } \\
\hline Horizontal & 8 & None & None \\
\hline 1 Muscle & 1 & & \\
\hline 2 Muscles & 7 & & \\
\hline Vertical & 2 & NA & None \\
\hline 1 Muscle & 2 & & \\
\hline Vertical and horizontal & 1 & 1 & None \\
\hline \multicolumn{4}{|l|}{ Staged } \\
\hline Horizontal & 7 & 3 & 2 \\
\hline 1 Muscle & 5 & & 1 \\
\hline 2 Muscles & 2 & & 1 \\
\hline Vertical & 2 & NA & None \\
\hline 1 Muscle & 1 & & \\
\hline 2 Muscles & 1 & & \\
\hline Vertical and horizontal & 2 & 2 & None \\
\hline
\end{tabular}

«Three patients not available for day 1 ICG study; tone patient not available for day 1 ICG study; $\mathrm{NA}=$ patients not available for day 1 ICG study.

Preoperative ICG angiography was taken within 1 week before surgery. Early postoperative angiography was taken within 1 week after surgery. The mean follow up by late ICG iris angiogram was 8.6 weeks and ranged from 3 to 22 weeks after surgery. Sectorial filling delay was defined as a 10 second filling delay of over one or more clock hours. The development of adverse reactions to ICG was recorded.

\section{Results}

Preoperative strabismus diagnoses included 18 eyes $(40 \%)$ of non-paralytic origin, $14(31.1 \%)$ paralytic, nine (20\%) restrictive, and four $(8.9 \%)$ others. There were 24 female and 21 male patients with an age range of 15-79 years (mean age 54.6 years). Twenty three patients had primary rectus surgery and 11 patients had secondary rectus surgery. Eleven patients had staged rectus surgery.

The ICG iris angiogram findings are displayed in Table 1.

Preoperative ICG iris angiography of 23 patients (nine brown irides, 10 blue, and four green) in the primary group served as control subjects. The arm to iris circulation time ranged between 17 and 22 seconds for the ICG dye to reach the iris vasculature after injection. The filling of the dye started at variable locations peripherally from the iris root; often the nasal aspect of the iris tended to fill first and the temporal aspect followed. The superior and inferior portions filled in the intermediate phase between the nasal and temporal filling. Subsequently, filling of the iris vessels near the pupillary margin was noted. The radial vessels were joined in an incomplete circumferential fashion around the collarette, as the minor arterial circle of the iris. Filling of the whole iris usually required 5-10 seconds. Iris colour did not affect the imaging capability of ICG angiography. Figure 1A showed the filling pattern of a normal subject with brown iris.
PRIMARY RECTUS MUSCLE SURGERY (TABLE 1) In the horizontal rectus surgery group, four patients underwent single rectus muscle surgery, two medial (MR), and two lateral recti (LR) respectively. Five patients had recession and resection of one horizontal rectus. In comparing the preoperative ICG angiograms with the early and late postoperative angiograms, no appreciable difference in perfusion was identified in any of the eyes.

In the vertical group, three patients had only superior rectus (SR) surgery and another four had inferior rectus (IR) surgery. Two patients had combined SR and IR surgery. Six of the nine patients were noted to have early postoperative delayed iris filling (mean delay filling time 38 seconds, range $24-56$ seconds) which corresponded to the vertical recti surgery.

Figure 1B shows delayed filling of the superotemporal quadrantic and is present after SR surgery. The remaining iris demonstrated a normal filling pattern. Ten weeks after SR surgery, ICGA still shows persistent delay in filling in the superior quadrant.

Figure 1C demonstrates a delayed filling in the inferior temporal quadrant after recession of the IR, and was found in two patients. No delay in filling was noted in late postoperative photographs.

Two patients demonstrated a delayed filling in the corresponding superior and inferior segments of the iris (Fig 1D) after combined SR and IR disinsertion. The iris was completely filled in late frames at 6 months.

Five patients had combined adjacent vertical and horizontal recti surgery. One patient failed to have early ICG angiogram. The remaining four patients showed delay in the vertical segmental quadrant of the operated rectus muscle in early postoperative angiograms but nine of them showed persistent perfusion defects in late angiograms.

Figure $1 \mathrm{E}$ shows delayed filling is present in the superotemporal quadrant after combined SR and MR surgery, but no significant delay of the filling of the medial quadrant was noted. Five months postoperatively complete reperfusion of the iris in all quadrants was seen.

SECONDARY RECTUS MUSCLE SURGERY (TABLE 1) In the horizontal group, two patients had LR surgery and six patients had combined LR and MR surgery. None of these patients developed delay in filling in the early postoperative period.

In the vertical group, one patient had SR and the other one had IR surgery. Neither had early postoperative angiography, and their late angiography did not show any delay in filling. One patient (aged 58) who underwent IR and LR surgery had previous MR and LR surgery 5 months earlier. Early postoperative angiogram showed delay in filling over the corresponding inferior and temporal segments but nasal perfusion was normal (Fig 1F).

STAGED SURGERY OF THE RECTI MUSCLES (TABLE 1)

In the horizontal group, two patients had MR surgery years after previous rectus muscle sur- 

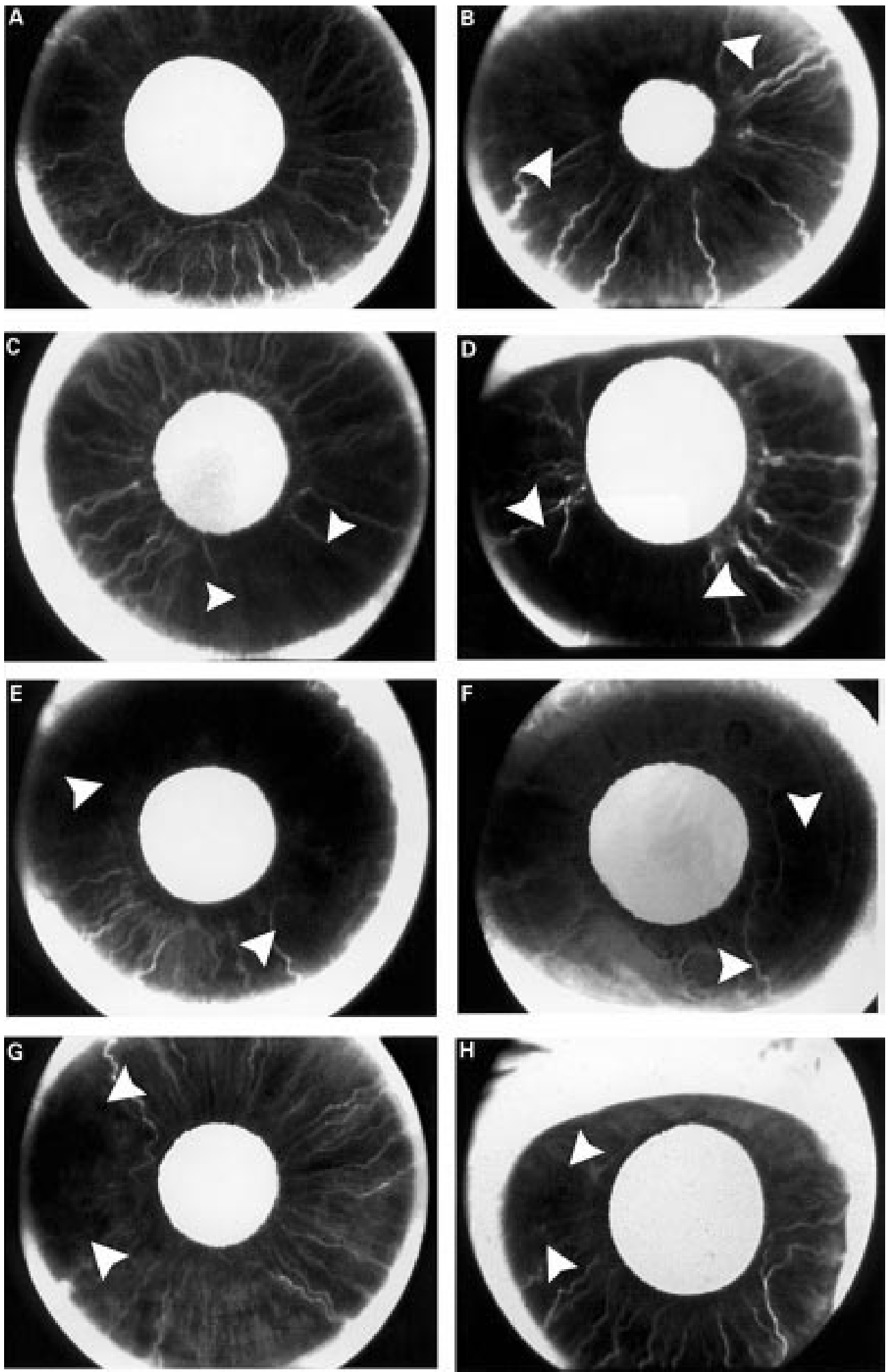

Figure 1 (A) Normal dark brown iris ICGA of the right eye in a 49 year old man. Late frame at 46 seconds showed complete filling of the iris. (B) ICGA of the right eye in a 68 year old man. One day after primary SR surgery. Superior and temporal sectors (arrowheads) filled out of phase by 34 seconds. (C) ICGA of the left eye in a 52 year old man. One day after primary IR surgery. The nasal, superior, and temporal sectors started to fill first which was followed by a delay in filling in inferior quadrant (arrowheads) by 40.2 seconds. (D) ICGA of the right eye in a 55 year old woman. One day after primary SR and IR surgery. Superior and inferior temporal sectors (arrowheads) showed delay in perfusion by 25 seconds. (E) ICGA of the left eye in a 59 year old man. One day after primary SR and MR surgery. Persistent superior sector delay (arrowheads) by 6 minutes; no nasal sector delay in filling. (F) ICGA of the left eye (previous 5 month history of $L R$ and MR surgery) in a 58 year old woman. One day after IR and LR surgery. Inferior and temporal sectors filled out of phase (arrowheads). Nasal sector perfusion was normal. (G) ICGA of the right eye (previous 3 year history of MR surgery) in a 81 year old man. Preoperative ICGA showed persistent temporal sector delay at late frames. Change of surgical plan and limited surgery to LR only in this eye. One day after LR surgery. Temporal sector delay (arrowheads) persisted at 38.8 seconds. (H) ICGA of the right eye (previous 18 month history of SR and IR surgery) in a 15 year old girl. One day after MR and LR surgery. Temporal sector delay (arrowheads) in filling of 27.9 seconds and nasal sector was well perfused at early frames because of the MR anterior ciliary artery preservation technique 2 months after surgery. 
gery. One (aged 43) had previous vertical recti transposition performed 4 years earlier and the other patient had SR surgery 2 years earlier. Neither demonstrated delay in iris filling preoperatively. One of the three patients who had LR surgery, a 40 year old man with previous vertical recti transposition surgery and one of the two who had combined LR and MR surgery after previous MR surgery, showed no delay in filling postoperatively.

However, three patients in the horizontal group did show signs of filling delay. The first patient (aged 59) had LR surgery following previous surgery of three other recti surgery 7 months earlier and developed a temporal filling defect. The second patient (aged 81) had LR resection and has had MR surgery 3 years earlier. This patient showed a temporal filling defect before surgery (Fig 1G). Because of this preoperative finding, the original plan of $M R$ surgery was changed instead to LR. The third patient (aged 15) had a history of combined SR and IR surgery 18 months previously. The patient then had combined LR and MR procedure (with ciliary artery sparing technique performed). She continued to show temporal delay in both early and late frames postoperatively (Fig 1H).

Two patients in the vertical group did not have early postoperative frames but had normal late frames.

In the combined group one patient (aged 58), with MR and LR surgery 5 months previously, underwent staged/secondary LR and IR surgery. He developed inferior and temporal defect in the early postoperative frame, but was lost to follow up for the late ICG angiogram. Another patient who underwent staged SR and LR (aged 34, had MR surgery 18 years earlier) showed temporal filling defect postoperatively.

None of the patients in the study had developed any clinical sign of anterior segment ischaemia postoperatively.

\section{Discussion}

ICG angiography did not demonstrate any delay in filling after primary horizontal surgery, but did demonstrate filling defects in the immediate postoperative period in $57 \%$ (four out of 14 patients did not have immediate postoperative ICG angiograms) of both primary vertical and combined vertical/ horizontal. Similar findings were observed as reported by Hayreh and Scott ${ }^{7}$ and Olver and Lee $^{89}$ with fluorescein dye.

In the staged surgery group, ICGA showed delay in filling temporally 1 day after surgery in three out of four patients undergoing horizontal recti surgery after previous vertical rectus surgery. Two of these had previous combined SR and IR surgery. One of them (a 15 year old girl who had combined MR and LR surgery) developed a persistent filling defect in late phase but had no clinical sign of ASI. The other patient had a preoperative temporal filling defect after previous combined MR and IR surgery. The surgical plan was changed to a LR surgery instead of MR surgery in order to avoid potential non-perfusion of the nasal quadrant postoperatively. This patient showed a persistent filling defect temporally in late phase.

Two other patients (with previous history of horizontal recti surgery) in the combined vertical and horizontal recti group (one had SR and LR and the other had IR and LR surgery) also showed temporal delay in filling in the immediate postoperative period. None of our patients who underwent secondary or staged vertical surgery demonstrated delay in filling in ICG angiograms 6-8 weeks postoperatively.

In our study, patients in the secondary horizontal group did not demonstrate any persistent filling defect, confirming those observations. ${ }^{89}$ One patient in our study, who had combined IR and LR surgery, did demonstrate a persistent filling defect inferiorly. Hayreh and Scott ${ }^{7}$ reported a similar fluorescein filling defect in one patient.

Based on our cases with filling defects in the immediate postoperative phase, we found that vertical rectus surgery either performed individually or combined with horizontal rectus surgery, demonstrated significant delays in filling. But the iris circulation recovered well in the late postoperative phase. The filling defects in the early postoperative phase do not necessarily indicate ASI but may be considered a physiological response following rectus muscle surgery.

Based on our late ICG angiogram findings, 12 of 13 patients who showed delayed iris perfusion early after surgery had complete iris reperfusion a mean of 10.4 weeks postoperatively (range 3-22 weeks). We recommend that further strabismus surgery should be delayed for at least 12 weeks following surgery. If high risk factors are present, ICG or fluorescein iris angiogram should be considered before contemplating further rectus surgery. Olver and Lee $^{89}$ suggested that the safe interval before further rectus surgery should be at least 2 or 3 months.

There were three patients with dysthyroid eye disease in our study and none of them developed sign of delay of ICG filling angiographically or any clinical sign of ASI. Olver and Lee ${ }^{8}$ showed that primary vertical muscle surgery was the determining factor causing sector filling delay angiographically in six patients with Graves' ophthalmopathy.

Neither IFA ${ }^{10}$ nor ICG angiogram can predict which patients are likely to develop ASI postoperatively. These dyes are only able to demonstrate the degree of iris hypoperfusion preoperatively in order to allow the surgeon to change the surgical plan to try to avoid postoperative ASI.

In summary, ICG is a safe dye and is especially useful for displaying iris circulation in pigmented irides to detect perfusion changes pre-strabismus and post-strabismus surgery. It has a further advantage of being a protein bound molecule intravascularly which allows for very precise evaluation of iris hypoperfusion.

Proprietary interest: none.

1 Saunders RA, Phillips MS. Anterior segment ischaemia after three muscles surgery. Ophthalmology 1988;95:533-7. 
2 Kottow MH. Fundamentals of interpretation: normal angiogram. In: Anterior segment fluorescein angiography. Baltimore: Williams and Wilkins, 1978:51.

3 Hayreh SS, Scott WE. Fluorescein iris angiography, 1. Normal pattern. Arch Ophthalmol 1978;96:1383-9.

4 Cherrick GB, Stein SW, et al. Indocyanine green: observations on its physical properties, plasma decay, and hepatic extraction. $\mathcal{F}$ Clin Invest 1960;39:592-600.

5 Brucker AJ. Indocyanine green angiography (Editorial). Retina 1992;12:187.

6 Yannuzzi LA, Slaker JS Sorenson JA, et al. Digital indocyanine green videoangiography and choroidal neovascularization. Retina 1992;12:191-223.
7 Hayreh SS, Scott WE. Fluorescein iris angiography. 2. Disturbances in iris circulation following strabismus operation on the various recti. Arch Ophthalmol 1978;96:1390400 .

8 Olver JM, Lee JP. The effects of strabismus surgery on anterior segment circulation. Eye 1989;3:318-26.

9 Olver JM, Lee JP. Recovery of anterior segment circulation after strabismus surgery in adult patients. Ophthalmology 1992;99:305-15.

10 Saunders RA, Bluestein EC, Wilson E, et al. Anterior segment ischaemia after strabismus surgery. Surv Ophthalmol 1994;38:456-66.

Video Reports (www.bjophthalmol.com)

Capsule staining and mature cataracts: a comparison of indocyanine green and trypan blue dyes $D F$ Chang

Pearls for implanting the Staar toric IOL D F Chang 